\title{
Formation of $\mathrm{CeO}_{2}-\mathrm{ZrO}_{2}$ Solid Solution Nanocages with Controllable Structures via Kirkendall Effect \\ Supporting information
}

\section{Experimental Section}

Chemicals: All chemicals were of analytical grade and were used as received without further purification. Deionized water was used throughout. $\mathrm{Ce}\left(\mathrm{NO}_{3}\right)_{3} \cdot 6 \mathrm{H}_{2} \mathrm{O}, \mathrm{ZrOCl}_{2} \cdot 6 \mathrm{H}_{2} \mathrm{O}$, ethylene glycol were supplied by Beijing Chemical Reagent Company.

Synthesis of spherical ceria nanocrystal clusters: $1 \mathrm{ml} 0.5 \mathrm{M} \mathrm{Ce}\left(\mathrm{NO}_{3}\right)_{3}$ solution was added in $30 \mathrm{ml}$ glycol with stirring, then the mixture were sealed and treated at $180^{\circ} \mathrm{C}$ for $16 \mathrm{~h}$.

Synthesis of nearly ceria nanocrystal clusters: $1 \mathrm{ml} 0.5 \mathrm{M} \mathrm{Ce}\left(\mathrm{NO}_{3}\right)_{3}$ solution and $4 \mathrm{ml} \mathrm{H}_{2} \mathrm{O}$ were added in $30 \mathrm{ml}$ glycol with stirring, then the mixture was sealed and treated at $180{ }^{\circ} \mathrm{C}$ for $16 \mathrm{~h}$.

Synthesis of Ce-Zr-O nanocages: $0.3 \mathrm{ml} 0.5 \mathrm{M} \mathrm{ZrOCl}_{2}$ solution was added to the colloid ceria clusters obtained above. Then the mixture was sealed and heated at $180{ }^{\circ} \mathrm{C}$ for $8 \mathrm{~h}$. The $\mathrm{CeO}_{2} @ \mathrm{Ce}_{1-x} \mathrm{Zr}_{x} \mathrm{O}_{2}$ hollow core-shell structure were obtained by reducing the reaction time to $3 \mathrm{~h}$ and washed by ethanol with centrifugated at $5000 \mathrm{r} / \mathrm{min}$ for $5 \mathrm{~min}$ several times.

Transmission electron microscopy (TEM): The size and morphology of the Nanocrystals were determined by a JEOL JEM-1200EX transmission electron microscope (TEM) at $120 \mathrm{kV}$, and a Tecnai G2 F20 S-Twin high-resolution transmission electron microscope (HRTEM) at $200 \mathrm{kV}$. Samples of ceria CNCs were prepared by placing a drop of reaction solution on the surface of a copper grid; samples of ceria- zirconia nanocages were prepared by placing a drop of a dilute ethanol dispersion of nanocages on the surface of a copper grid. 
SI 1. Tem images of the monodispersed ceria spherical-like clusters with different magnifications.

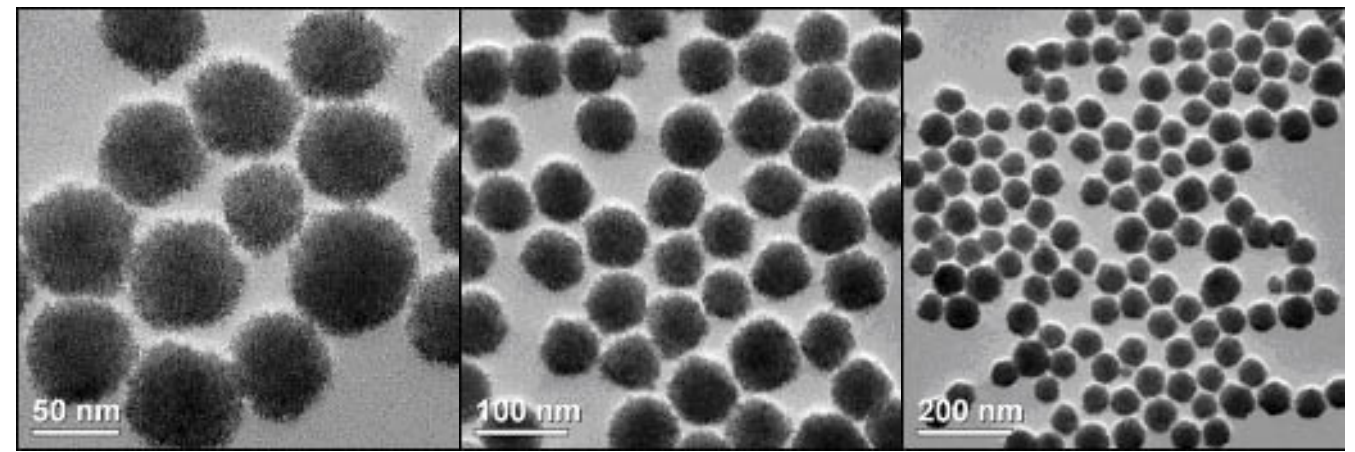

SI 2. HRTEM images of different parts of a single spherical-like ceria nanocrystal clusters.
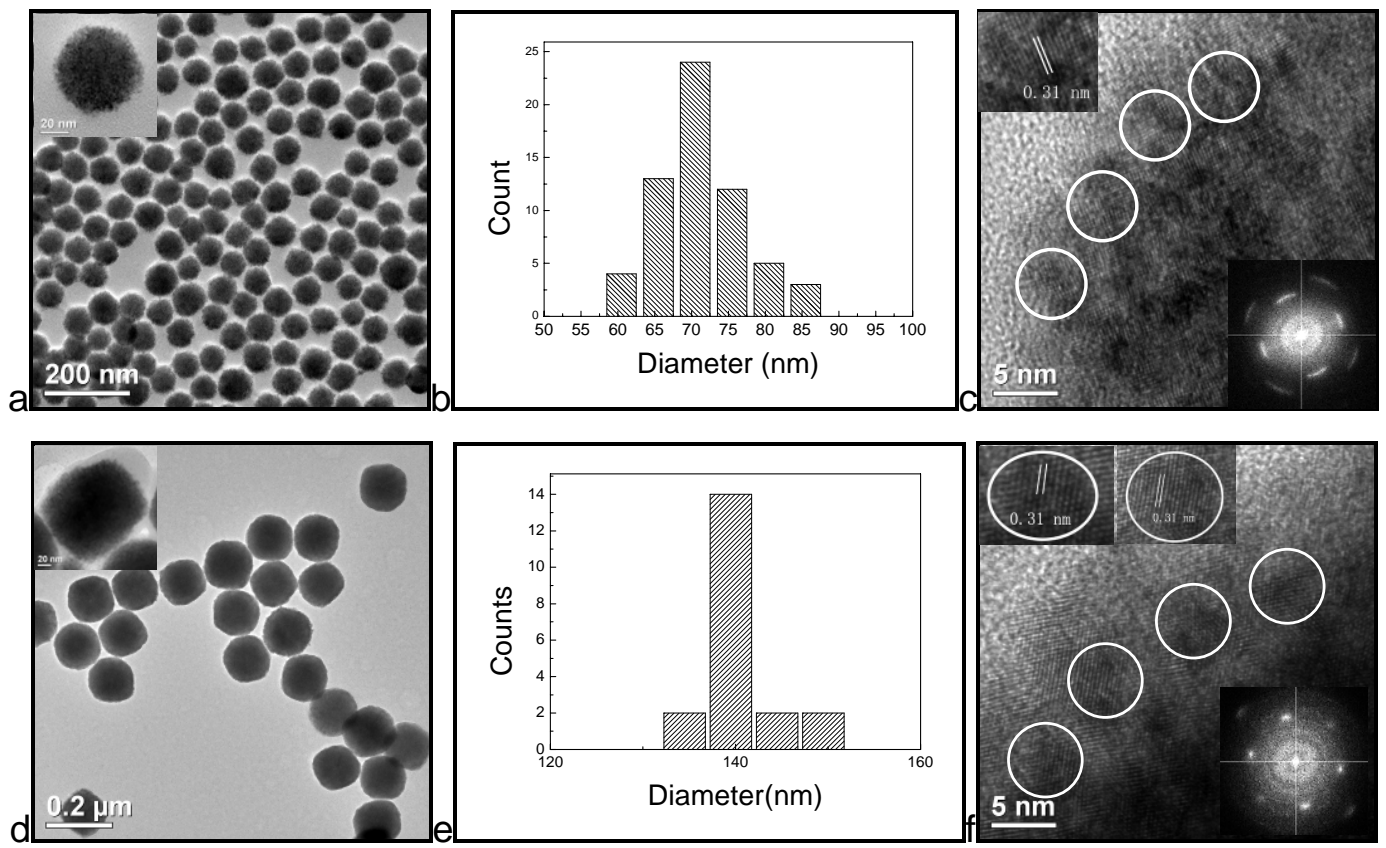

The water concentration in the reaction system has great influence on the morphology of the obtained ceria. When $4 \mathrm{ml} \mathrm{H}_{2} \mathrm{O}$ was added in the former system, nearly cubic-like clusters have been formed. The typical TEM image shown in figure $1 \mathrm{~d}$ revealed the clusters are of narrow size distribution and more regular shape. Based on carefully observation on the HRTEM images (figure. 1f), it can be concluded that the big cluster was also comprised of many small particles with exposed $\{111\}$ crystal planes and the particle size of the primary small particles is similar to that of the spherical-like clusters. However, the crystal fringes in the cubic-like cluster seem to be more continuous and the space between two adjacent small particles becomes smaller compared to that of spherical-like clusters. In the condition of high water concentration in reaction system, the adsorption of $\mathrm{H}_{2} \mathrm{O}$ on surface of the small particles increased and the adsorption of $\mathrm{HOCH}_{2} \mathrm{CH}_{2} \mathrm{OH}$ on the surface decreased due to the increase of water concentration and the competitive adsorption of water on the surface of small particles with 
glycol. As a result, the small particles more readily aggregated with each other due to the decreasing protect effect of glycol. In this condition, the primary small particles orientedly attached with each other more closely and aligned with the adjacent one more orderly according to crystallographics, which leaded to the more regular morphology compared to spherical ones.

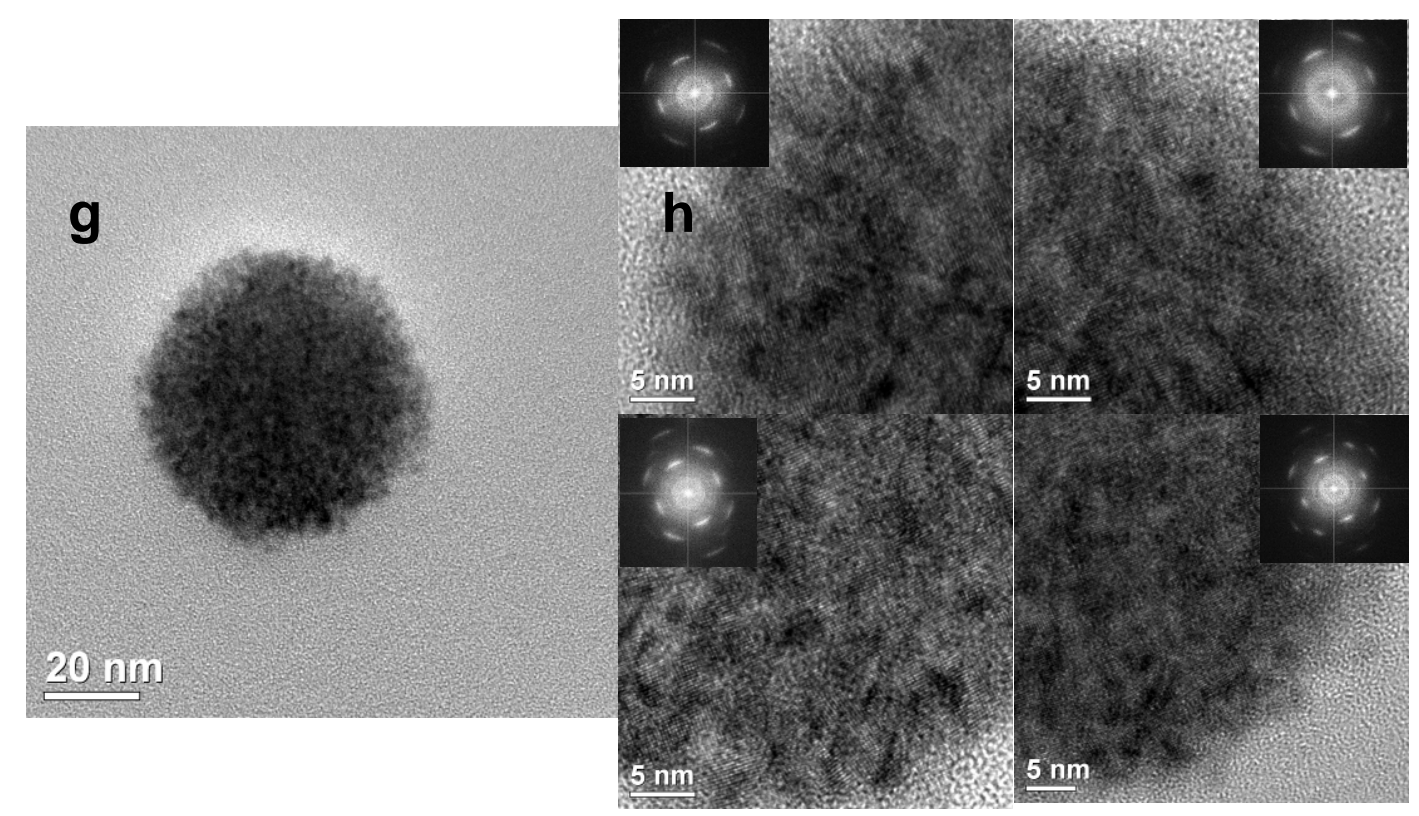

Figure $g$ shows a single spherical-like nanocrystal cluster. Figure $h$ show the HRTEM images of the different parts of the single cluster. The inserts are the corresponding fourier transformation electron diffraction patterns. It can be seen form the inserts that the diffraction patterns are neither the typical polycrystal rings, nor the typical single crystal dots. The patterns here are elongated dots, which implied the big cluster are comprised by many small nanocrystals which oriented attached with each other on similar direction. What's more, it is similar that the orientation of the fourier transformation electron diffraction patterns of different parts of the single cluster, which revealed that the primary small particles crystallographically aligned with each other orderly in the whole cluster. This phenomenon can be well understood as the result of oriented attachment of primary small particles and subsequent high-temperature sintering during synthesis ${ }^{[20]}$ 
SI 3. Energy dispersive spectrometry of a single hollow sphere.

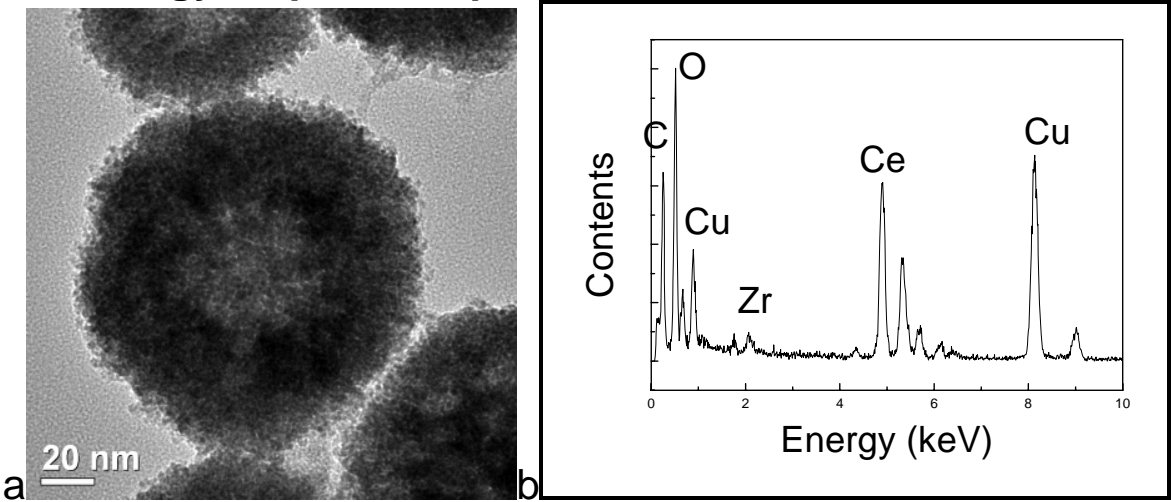

c

\begin{tabular}{|l|l|l|l|l|}
\hline Element & Weight & Atomic \% & Uncertainty\% & Correction \\
\hline $\mathrm{O}(\mathrm{K})$ & 42.412 & 85.504 & 1.437 & 0.514 \\
\hline $\mathrm{Zr}(\mathrm{L})$ & 10.041 & 3.550 & 0.730 & 0.877 \\
\hline $\mathrm{Ce}(\mathrm{K})$ & 47.545 & 10.944 & 3.746 & 0.863 \\
\hline
\end{tabular}

a) TEM image of hollow nanospheres which were synthesized at the molar ratio of $\mathrm{Zr}^{4+} / \mathrm{CeO}_{2}$ is 3:10; b) the energy spectrum of a whole hollow nanosphere; c) the calculation of the element content in the hollow nanosphere.

It can be concluded from the calculation result that the molar ratio of $\mathrm{Ce} / \mathrm{Zr}$ ratio is similar with the original molar ratio of the reactants $\mathrm{Zr}^{4+} / \mathrm{CeO}_{2}$. 


\section{SI 4. XRD patterns analysis}

X-ray powder diffraction patterns of the spherical (a) and nearly cubic-like ceria clusters (b), XRD patterns of the spherical nanocages (c) and nearly cubic-like nanocages (d); literature values for the peak positions and intensities for bulk ceria samples are indicated by the vertical bars (JCPDS no. 65-0859)

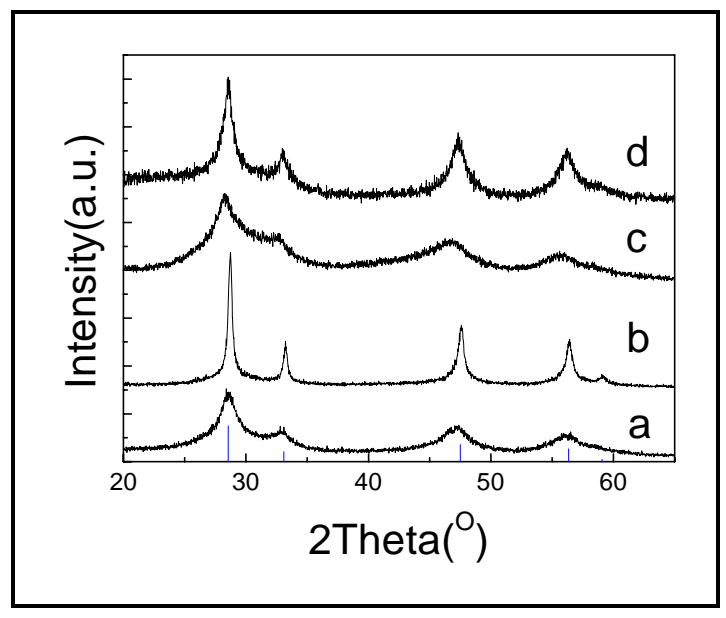

The XRD patterns of the spherical nanocages before and after annealing at $500^{\circ} \mathrm{C}$ for $2 \mathrm{~h}$

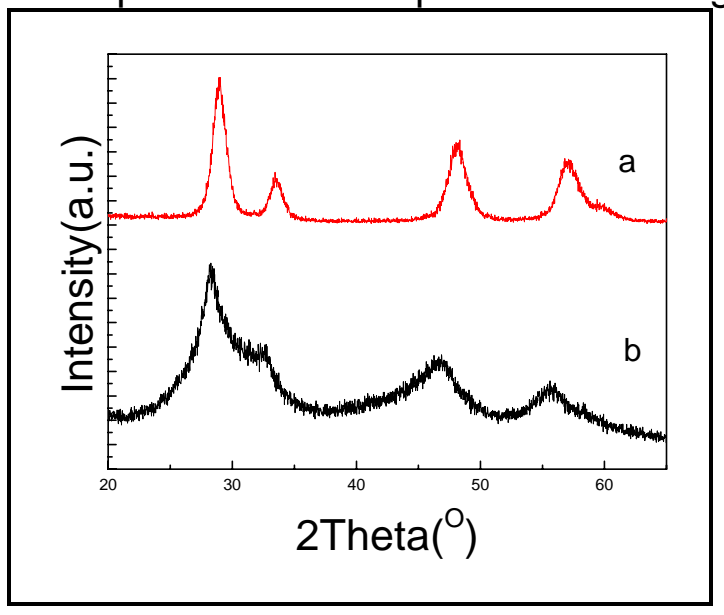




\section{SI 5. Tem images about the nanocages with different shell thickness}

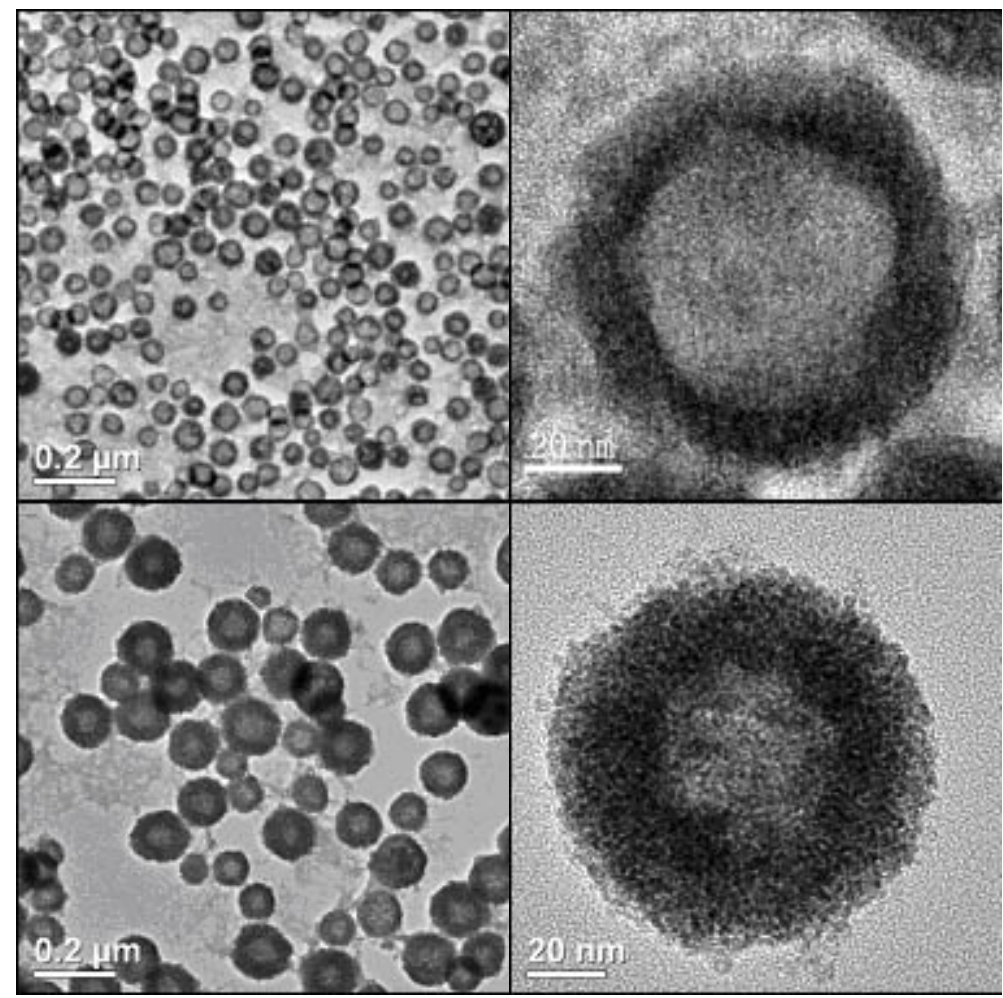

The shell thickness of the nanocages can be adjusted by tunning the synthetic parameters, such as the reaction time. The shell becomes thinner along with the increase of the reaction time.

SI 6. the transition state of the nearly cubic-like nanocages

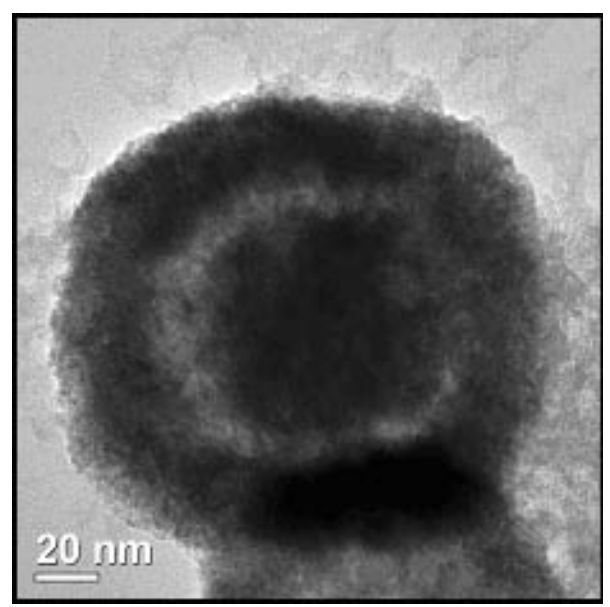




\section{SI 7. TEM images of the nanocages formed at different $\mathrm{Zr} / \mathrm{CeO}_{2}$ molar ratio}
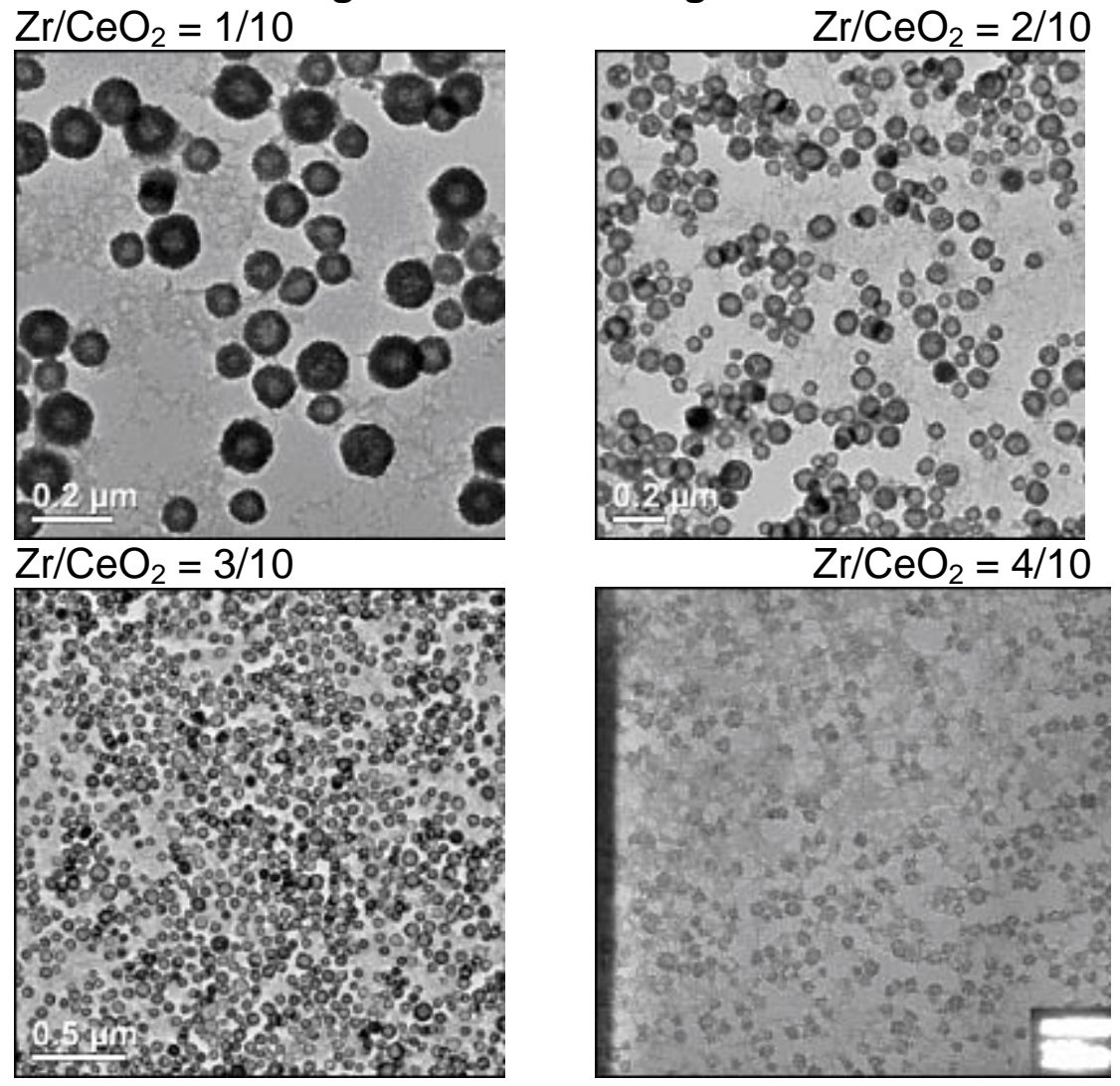

$\mathrm{Ce}_{1-x} \mathrm{Zr}_{x} \mathrm{O}_{2}$ nanocages with different composition $(x<0.5)$ have been successfully synthesized by adjusting the input of $\mathrm{Zr}^{4+}$ and the molar ratio of $\mathrm{Zr}^{4+} /$ ceria in the reaction system. However, when $x \geqslant 0.5$, there is only irregular nanoparticles of $\mathrm{Ce}_{1-x} \mathrm{Zr}_{x} \mathrm{O}_{2}$ formed. This is due to the relatively large difference between $\operatorname{Zr}(\mathrm{IV})$ and $\mathrm{Ce}(\mathrm{IV})$ ionic radii and the different crystal structures of $\mathrm{ZrO}_{2}$ and $\mathrm{CeO}_{2}$ at room temperature (monoclinic vs. face-centered cubic). When the molar ratio of $\mathrm{Zr}^{4+} /$ ceria increases, the crystal structure of $\mathrm{Ce}_{1-\mathrm{x}} \mathrm{Zr}_{x} \mathrm{O}_{2}$ evolves from cubic phase to tetragonal phase. The original ceria clusters can't act as the physical template due to the misfit of the crystal structure between the newly formed $\mathrm{Ce}_{1-x} \mathrm{Zr}_{x} \mathrm{O}_{2}$ (tetragonal phase) and the origin ceria clusters (cubic phase), which finally leads to the collapse of the nanocrystal clusters and no hollow nanostructures form. Oppositely, when $\mathrm{x}$ is low, the crystal structure of the formed $\mathrm{Ce}_{1-\mathrm{x}} \mathrm{Zr}_{\mathrm{x}} \mathrm{O}_{2}$ is also face-centered cubic phase. The crystal structure matching of $\mathrm{Ce}_{1-\mathrm{x}} \mathrm{Zr}_{x} \mathrm{O}_{2}$ and ceria make the original monodispersed ceria clusters a good physical template for $\mathrm{Ce}_{1-x} \mathrm{Zr}_{x} \mathrm{O}_{2}$ growth. Thus, $\mathrm{Ce}_{1-x} \mathrm{Zr}_{x} \mathrm{O}_{2}$ nanocages with uniform size, well defined shapes, and tunable compositions can be obtained. 


\section{SI 8. The HRTEM images of the spherical $\mathrm{Ce}_{0.7} \mathrm{Zr}_{0.3} \mathrm{O}_{2}$ nanocages}

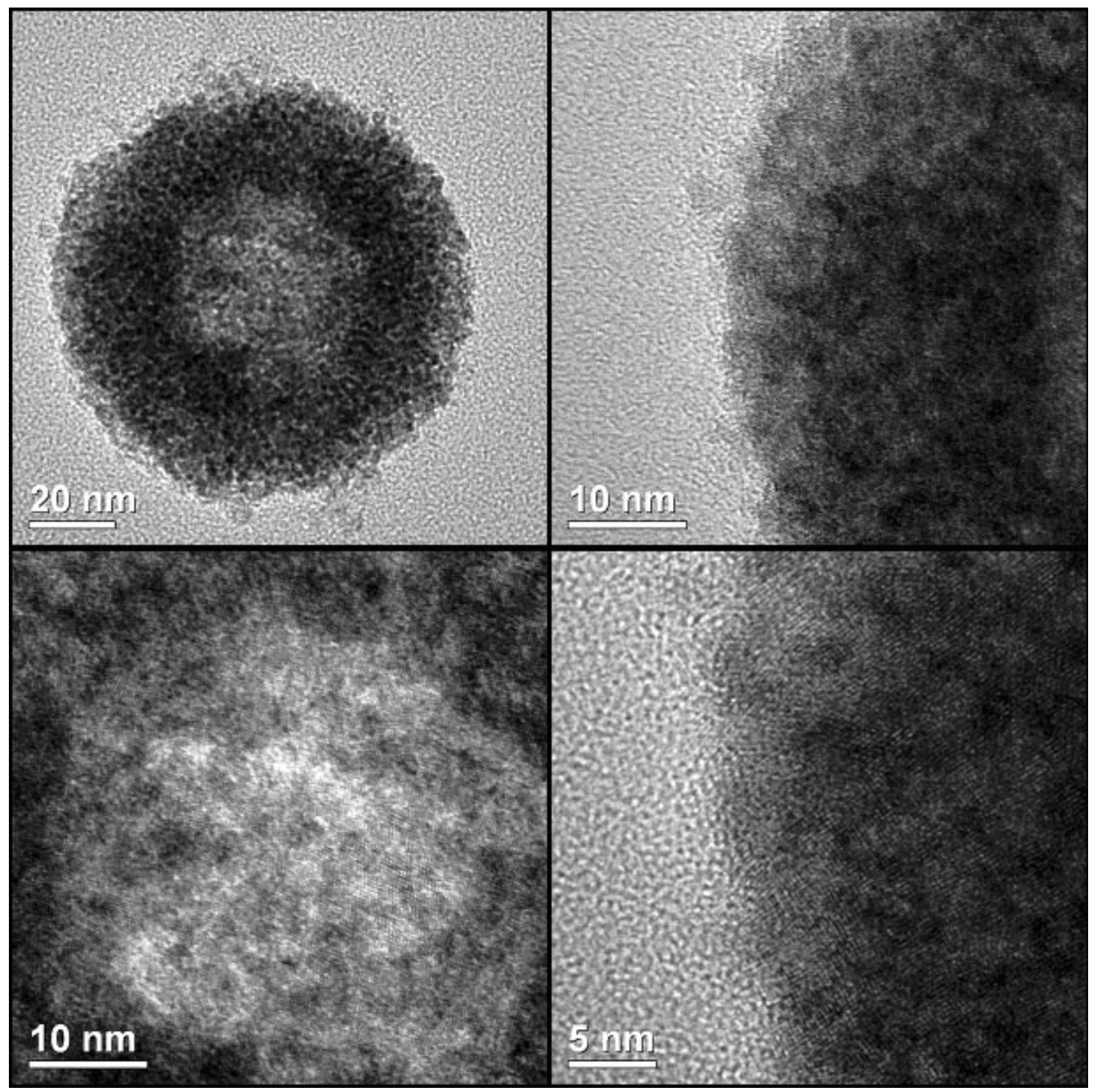

The HRTEM images of a single spherical $\mathrm{Ce}_{0.7} \mathrm{Zr}_{0.3} \mathrm{O}_{2}$ nanocage show clear crystal fringes, which reveal the nanocage was crystalline. Similar with the $\mathrm{CeO}_{2}$ clusters, the $\mathrm{Ce}_{0.7} \mathrm{Zr}_{0.3} \mathrm{O}_{2}$ nanocage was also comprised of many small nanocrystals. 


\section{SI 9. Infrared spectrum of spherical $\mathrm{Ce}_{0.7} \mathrm{Zr}_{0.3} \mathrm{O}_{2}$ nanocages}

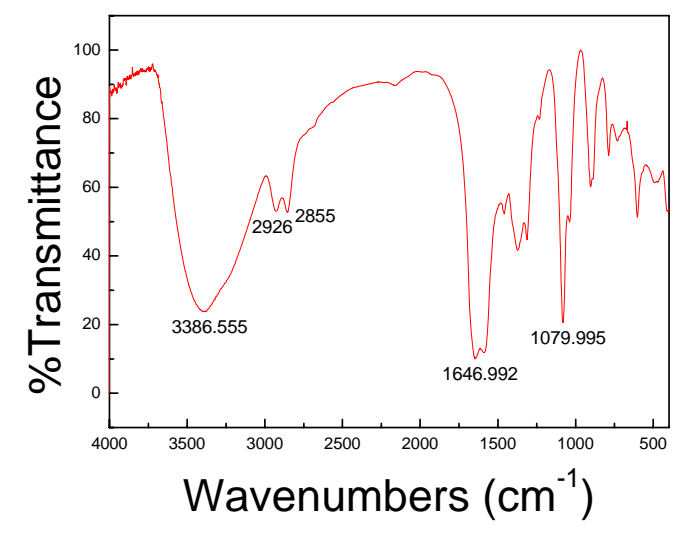

The wide band at $3000-3600 \mathrm{~cm}^{-1}$ is assigned to $\mathrm{O}-\mathrm{H}$ vibrations. The sharp bands at $\sim 2948 \mathrm{~cm}-1$ are assigned to the asymmetric and symmetric methylene stretching modes. The sharp bands at $\sim 1079 \mathrm{~cm}-1$ are assigned to the $\mathrm{C}-\mathrm{O}$ stretching vibration of alcohol. These characteristic bands can confirm the existence of glycol. The bands at $\sim 1650 \mathrm{~cm}^{-1}$ can be assigned to the $\mathrm{C}=\mathrm{O}$ vibrations, which implied that there is partly oxidation of glycol in the reaction process.

Based on the FTIR spectra, glycol is believed to coat the surface of the as-prepared samples. This result can explain the easiness of dispersion of the as-prepared samples in polar solvents. 


\section{SI 10. the statistics date of the diameter before and after the reaction}
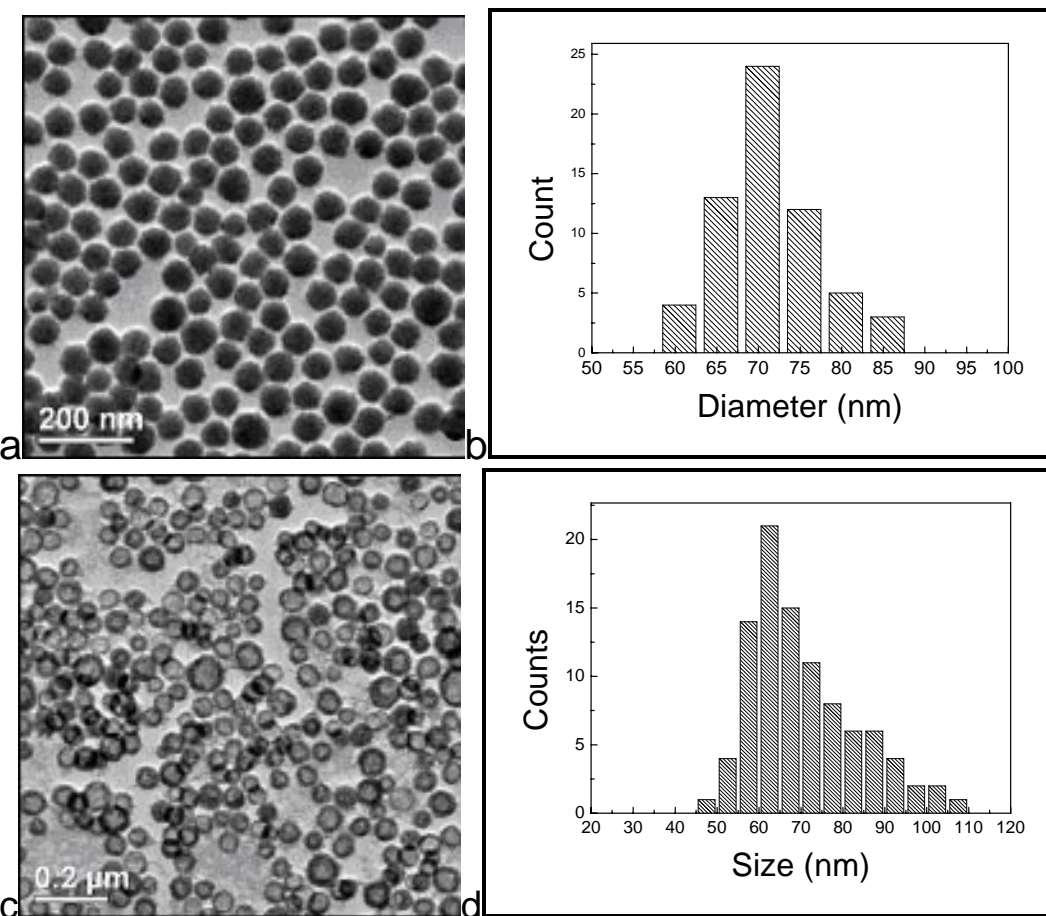

a: TEM image of the $\mathrm{CeO}_{2}$ clusters before the reaction; $b$ : the corresponding size distribution

c: TEM image of the $\mathrm{Ce}_{0.7} \mathrm{Zr}_{0.3} \mathrm{O}_{2}$ hollow spheres after reaction; $\mathrm{d}$ : the corresponding size distribution

From the statistics size distribution before and after the reaction, It can be seen that the average size after the reaction is similar with the average size before the reaction. 


\section{SI 11. The EDS analysis of the $\mathrm{CeO}_{2} @ \mathrm{Ce}_{x} \mathrm{Zr}_{1-\mathrm{x}} \mathrm{O}_{2}$ hollow core-shell strucutre}
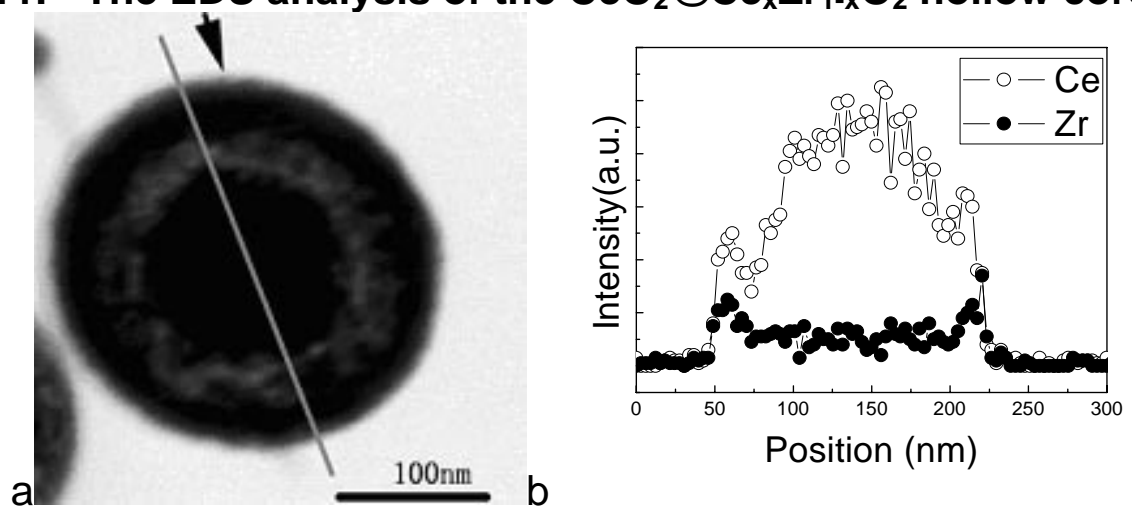

$\mathrm{a}$, the bright field image of a single $\mathrm{CeO}_{2} @ \mathrm{Ce}_{x} \mathrm{Zr}_{1-\mathrm{x}} \mathrm{O}_{2}$ hollow core-shell nanostructure; the gray line is the EDS line scanning route; b: compositional line profile across the nanostructure probed by EDS line scanning.
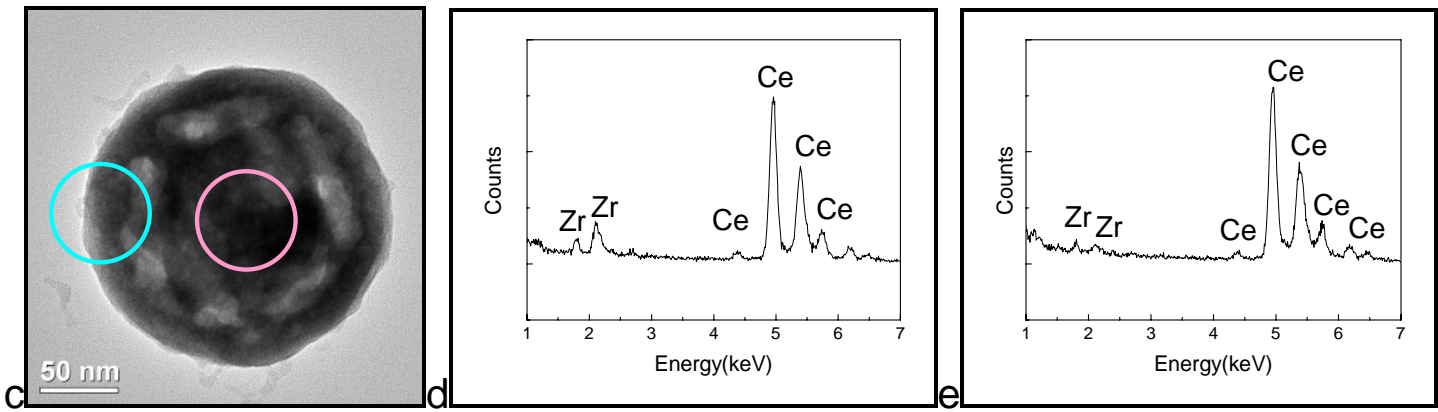

c) high magnification TEM images of a single sphere; d) the energy spectrum of the shell of the sphere (as the green circle marked);e) the energy spectrum of the center area of the sphere (the pink circle marked) 